\title{
Facilitating Biomimetic Syntheses of Borrerine Derived Alkaloids by Means of Flow-Chemical Methods
}

\author{
Sonja B. Kamptmann ${ }^{\mathrm{A}}$ and Steven V. Ley ${ }^{\mathrm{A}, \mathrm{B}}$ \\ ${ }^{A}$ Department of Chemistry, University of Cambridge, Lensfield Road, Cambridge CB2 1EW, United \\ Kingdom \\ ${ }^{\mathrm{B}}$ Corresponding author. Email: svl1000@cam.ac.uk
}

Dedicated to the memory of Kappa Cornforth, a great scientist and human being.

\begin{abstract}
Flow chemistry, although now commonly used for general synthetic chemistry, has not been applied extensively in a biomimetic fashion. Here we show how the flow syntheses of borrerine derived alkaloids can use these principles to obtain complex architectures in a single step.
\end{abstract}

In many examples described in the literature flow synthesis offers significant advantages compared to classical batch methods with regards to safety ${ }^{[1-4]}$ and environmental impact (e.g. through reduction of downstream-processing, lower energy consumption, lower solvent use), ${ }^{[5]}$ and allows reactions to be performed under a broader range of reaction conditions. ${ }^{[6-9]}$ Furthermore, flow chemistry often allows a more rapid reaction optimisation, ${ }^{[10-12]}$ and the use of in-line tools enables a better analysis of reaction pathways. ${ }^{[13,14]}$ Consequently, flow chemistry is now widely used in synthetic chemistry ${ }^{[15]}$ and has moreover increasingly applied in the area of complex natural product synthesis. ${ }^{[16-18]}$ Also, the many options to follow reactions via in-line analysis allows researcher to harness reactive intermediates in a controlled fashion, making flow chemistry an ideal tool for conducting biomimetic syntheses. However, only a few examples to date have been reported using flow chemistry to explore biomimetic pathways. ${ }^{[19-21]}$ Consequently, we have recently begun a program aimed at the generation of molecular complexity using flow-chemical methods to mimic the way a cell produces a compound, rather than in typical round-bottom flasks. First attempts of our group to realise biomimetic syntheses of natural products in flow proved to be very successful, demonstrating the predominance of flow reactions over batch reactions in cases where reactive intermediates are formed during the reaction. ${ }^{[21]}$ This has encouraged us to look at even more complex structures of which the borrerine derived alkaloids caught our attention (see fig. 1). The bisindole alkaloids borreverine (4) and isoborreverine (2) were first isolated from Borreria verticillata in the 1970 s. $^{[22,23]}$ In 1978 it was shown that the two molecules can be directly derived from the dimerisation of the naturally occuring indole alkaloid borrerine (1). ${ }^{[24]}$ Since that time a number of structurally related bisindole alkaloids have been isolated which are proposed to have a common biosynthetic origin derived from borrerine (1). ${ }^{[25-27]}$ These compounds are of particular interest since many of them have shown significant antimalarial activity, significantly even in chloroquine resistant strains of Plasmodium falciparum. ${ }^{[28-30]}$

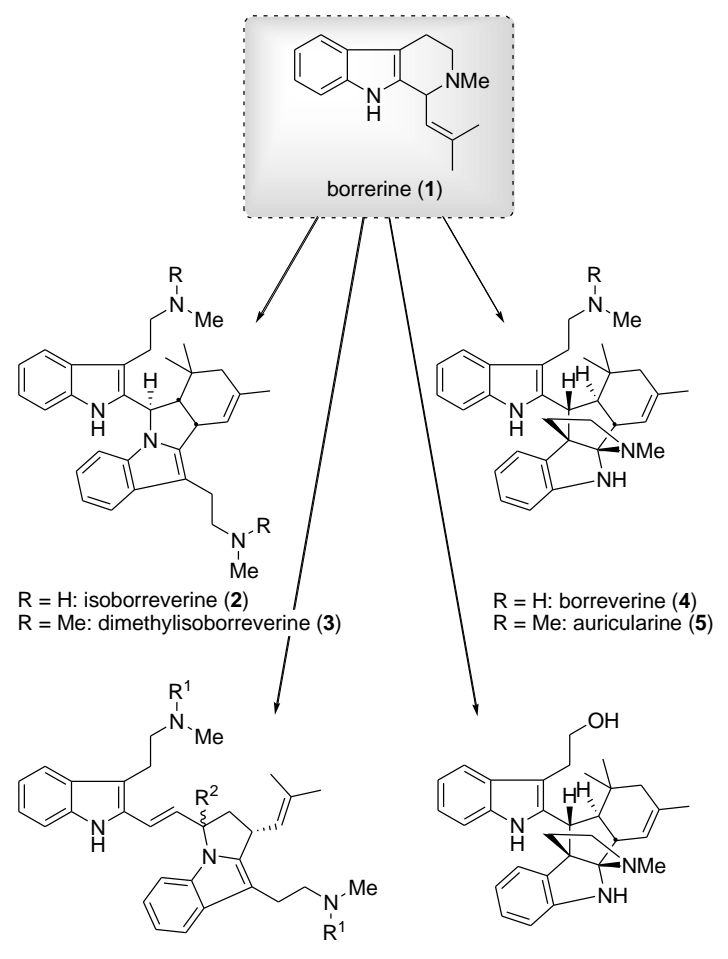

$\mathrm{R}^{1}=\mathrm{H}, \mathrm{R}^{2}=\alpha$-Me: flinderole $\mathrm{A}(\mathbf{6})$

$\mathrm{R}^{1}=\mathrm{Me}, \mathrm{R}^{2}=\alpha$-Me: flinderole $\mathrm{B}(\mathbf{7})$

spermacoceine (10)

$\mathrm{R}^{1}=\mathrm{H}, \mathrm{R}^{2}=\beta$-Me: desmethylflinderole $(\mathbf{8})$

$R^{1}=$ Me, $R^{2}=\beta$-Me: flinderole C $(9)$

Fig. 1. Borrerine derived alkaloids.

The isolation publications of these compounds were rapidly followed by relevant synthetic studies. ${ }^{[31-36]}$ Nevertheless, these syntheses were accompanied by issues such as a large number of synthetic steps, 
difficulties in up-scaling the reactions or the necessity for extensive purification steps to separate complex product mixtures, respectively.

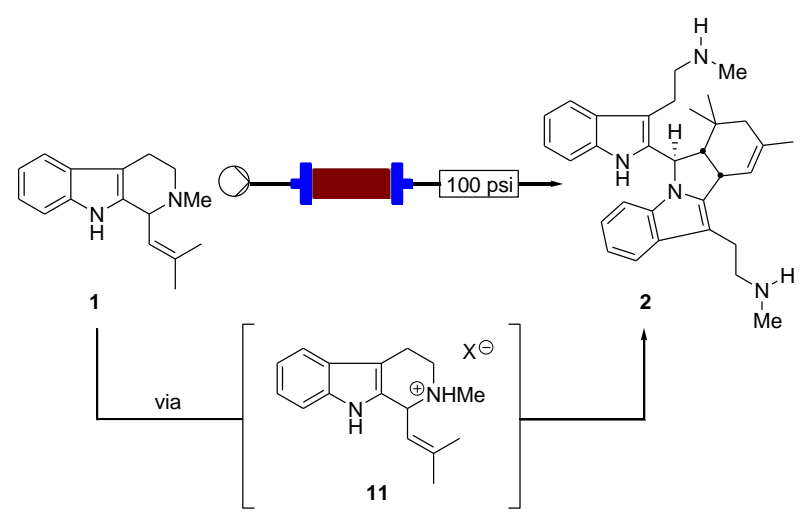

\begin{tabular}{|c|c|c|c|c|c|}
\hline Entry & Reagent & Solvent & $\mathrm{T}$ & $\begin{array}{c}\mathrm{FR} \\
{[\mathrm{ml} / \mathrm{min}]}\end{array}$ & $\begin{array}{c}\text { 1:11:2 } \mathbf{2}^{\mathrm{a}} \\
\text { (Yield 2) }\end{array}$ \\
\hline 1 & Amberlyst-Yb & $\mathrm{PhCH}_{3}$ & $150^{\circ} \mathrm{C}$ & 0.2 & 100:0:0 \\
\hline 2 & Amberlyst-Sc & $\mathrm{PhCH}_{3}$ & $150^{\circ} \mathrm{C}$ & 0.2 & 100:0:0 \\
\hline 3 & Amberlyst-In & $\mathrm{PhCH}_{3}$ & $150^{\circ} \mathrm{C}$ & 0.2 & 100:0:0 \\
\hline 4 & Amberlyst-Yb & $\mathrm{CH}_{2} \mathrm{Cl}_{2}$ & $100^{\circ} \mathrm{C}$ & 0.2 & 100:0:0 \\
\hline 5 & Amberlyst-Sc & $\mathrm{CH}_{2} \mathrm{Cl}_{2}$ & $100^{\circ} \mathrm{C}$ & 0.2 & 100:0:0 \\
\hline 6 & Amberlyst-In & $\mathrm{CH}_{2} \mathrm{Cl}_{2}$ & $100^{\circ} \mathrm{C}$ & 0.2 & 100:0:0 \\
\hline 7 & Nafion NR50 ${ }^{\circledR}$ & $\mathrm{CH}_{2} \mathrm{Cl}_{2}$ & r. t. & 0.1 & 100:0:0 \\
\hline 8 & PVPP-BF ${ }_{3}$ & $\mathrm{CH}_{2} \mathrm{Cl}_{2}$ & r. t. & 0.5 & 0:100:0 \\
\hline 9 & PVPP-BF ${ }_{3}$ & $\mathrm{CH}_{2} \mathrm{Cl}_{2}$ & r. t. & 0.1 & 0:100:0 \\
\hline 10 & $\mathrm{PVPP}_{-\mathrm{BF}_{3}}$ & $\mathrm{CH}_{2} \mathrm{Cl}_{2}$ & $40^{\circ} \mathrm{C}$ & 0.1 & $0: 84: 16$ \\
\hline 11 & PVPP-BF ${ }_{3}$ & $\mathrm{CH}_{2} \mathrm{Cl}_{2}$ & $70^{\circ} \mathrm{C}$ & 0.5 & $0: 81: 19$ \\
\hline 12 & PVPP-BF ${ }_{3}$ & $\mathrm{CH}_{2} \mathrm{Cl}_{2}$ & $70^{\circ} \mathrm{C}$ & 0.1 & $0: 75: 25$ \\
\hline 13 & PVPP-BF 3 & $\mathrm{CH}_{2} \mathrm{Cl}_{2}$ & $100^{\circ} \mathrm{C}$ & 0.5 & $0: 66: 34$ \\
\hline 14 & PVPP-BF ${ }_{3}$ & $\mathrm{CH}_{2} \mathrm{Cl}_{2}$ & $100^{\circ} \mathrm{C}$ & 0.2 & $0: 40: 60$ \\
\hline 15 & $\mathrm{PVPP}^{-\mathrm{BF}_{3}}$ & $\mathrm{CH}_{2} \mathrm{Cl}_{2}$ & $100^{\circ} \mathrm{C}$ & 0.1 & $\begin{array}{c}0: 0: 100 \\
(68 \%)\end{array}$ \\
\hline $16^{\mathrm{b}}$ & PVPP-BF ${ }_{3}$ & $\mathrm{CH}_{2} \mathrm{Cl}_{2}$ & $100^{\circ} \mathrm{C}$ & 0.5 & $0: 54: 46$ \\
\hline $17^{\mathrm{b}}$ & PVPP-BF ${ }_{3}$ & $\mathrm{CH}_{2} \mathrm{Cl}_{2}$ & $100^{\circ} \mathrm{C}$ & 0.3 & $0: 66: 34$ \\
\hline $18^{\mathrm{b}}$ & PVPP-BF ${ }_{3}$ & $\mathrm{CH}_{2} \mathrm{Cl}_{2}$ & $100^{\circ} \mathrm{C}$ & 0.2 & $0: 47: 53$ \\
\hline 19 & PVPP-BF ${ }_{3}$ & $\begin{array}{c}\mathrm{CH}_{2} \mathrm{Cl}_{2} / \\
\operatorname{DCE}(1: 1)\end{array}$ & $100^{\circ} \mathrm{C}$ & $0.1^{\mathrm{c}}$ & $\begin{array}{c}0: 0: 100 \\
(95 \%)\end{array}$ \\
\hline 20 & Silica-BF 3 & $\mathrm{CH}_{2} \mathrm{Cl}_{2}$ & r. t. & $0.5^{\mathrm{d}}$ & $\begin{array}{c}0: 0: 100 \\
(94 \%)\end{array}$ \\
\hline
\end{tabular}

Table 1. Optimisation of dimerisation of borreverine (1) to isoborreverine (2). [a] Determined by ${ }^{1} \mathrm{H}$ NMR; [b] $\mathrm{c}=0.008 \mathrm{M}$; all other experiments: $\mathrm{c}=0.04 \mathrm{M}$. Entry 8-19: $\mathrm{X}^{-}=\mathrm{BF}_{4}$. [c] Volume of the packed bed: $2.4 \mathrm{~mL}$, residence time ca. $30 \mathrm{~min}$. [d] Volume of the packed bed: $2.2 \mathrm{~mL}$, residence time ca. $3 \mathrm{~min}$.

We therefore turned to flow chemistry to avoid some of these problems. ${ }^{[37]}$ Firstly, we focused on the preparation of isoborreverine (2) which is a Diels-Alder product of the diene resulting from a ring opening of borrerine (1). As shown by Vallakati and May ${ }^{[33]} \mathbf{2}$ can be obtained by a acid-promoted one-step procedure from borrerine (1). We screened different solid-supported Lewis acids under different conditions (see table 1). No conversion was observed with rare earth based solid-supported Lewis acids such as Amberlyst-Yb, Amberlyst-Sc or Amberlyst-
$\operatorname{In}^{[38]}$ nor with Nafion NR50 ${ }^{\circledR}$ (entry 1-7). Success however came by using PVPP- $\mathrm{BF}_{3},{ }^{[39,40]}$ a polymersupported $\mathrm{BF}_{3}$ reagent (entry 8-19). After a quick optimisation study of the reaction conditions we found conditions which gave excellent conversion to isoborreverine (2), essentially without any side product formation. However, when $\mathrm{CH}_{2} \mathrm{Cl}_{2}$ was used as a solvent we isolated only $68 \%$ of the product, presumably because some of the product was stuck to the solid-supported Lewis acid. We therefore switched to a $\mathrm{CH}_{2} \mathrm{Cl}_{2} / 1,2$ dichloroethane (1:1, v:v) solvent mixture. A basic wash of the reaction mixture delivered isoborreverine (2) under these conditions as the free base in $95 \%$ yield (entry 19). With the more reactive silica-supported $\mathrm{BF}_{3}{ }^{[41]}$ (entry 20) we observed the selective dimerisation of $\mathbf{1}$ to $\mathbf{2}$ under considerably milder conditions and above all at higher flow rates. In each case the dimerisation product $\mathbf{2}$ was obtained in very good yield upon evaporation of the solvent. Since the NMR spectra showed only minor impurities there was no need for further purification prior to biological evaluation.

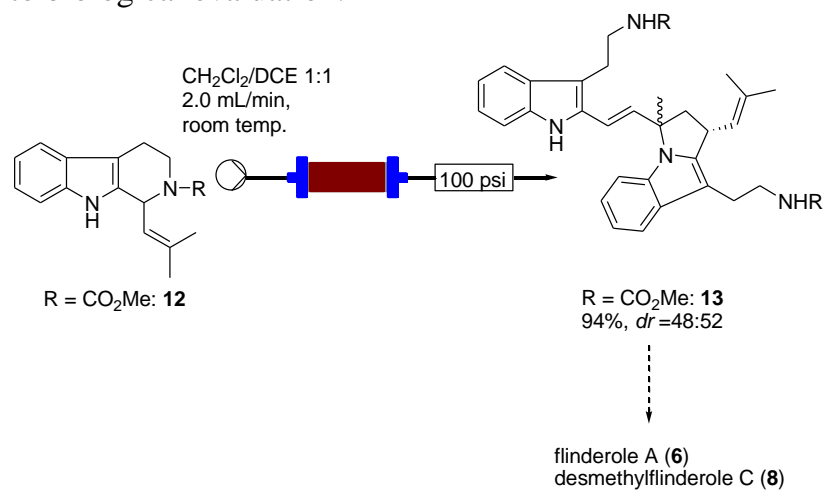

Fig. 2. Flow synthesis of $\mathbf{1 3}$ by dimerisation of $\mathbf{1 2}$. 13 is a precursor of flinderole A (6) and desmethylflinderole C (8). Volume of the packed bed: $2.4 \mathrm{~mL}$, residence time: ca. $2 \mathrm{~min}$.

With these promising results in hand we embarked on the synthesis of flinderole compounds 6 and 8 . These are formal [3+2] cylcloaddition products of the aforementioned ring-opened diene. We therefore studied the dimerisation of tetrahydropyridine- $N$-methylcarbamate protected borreverine $\mathbf{1 2}$ under similar conditions reported above for $\mathbf{2}$. As expected, dimerisation of $\mathbf{1 2}$ occured with even the less reactive $\mathrm{BF}_{3}$ reagent $\mathrm{PVPP}-\mathrm{BF}_{3}{ }^{[39,40]}$ under very mild conditions (room temperature) and at high flow rates $(2.0 \mathrm{~mL} / \mathrm{min})$. Compound $\mathbf{1 3}$, which is the carbamate-protected precursor of flinderole A (6) and desmethylflinderole C (8), was obtained in $94 \%$ yield as a diastereomeric mixture $\left(d r=48: 52^{[42]}\right)$ similar to previous syntheses.

In conclusion, this work and our previous study ${ }^{[21]}$ demonstrates that flow generated sequential complexity can be applied to biomimetic syntheses. The reactions can be optimised quickly and over a wide range of temperatures and pressures, as a result of the more controlled reaction conditions in flow. Furthermore, the system offers the opportunity to quickly obtain analogues of the respective natural products for further biological testing. Combining flow synthesis with bioassay platforms is already proving a successful strategy ${ }^{[43]}$ to expand the opportunities in the area. 


\section{Experimental}

General Experimental Procedure for the Flow Reactions $^{[37]}$

A solution of the substrate (preloaded in a $5 \mathrm{~mL}$ loop, $0.2 \mathrm{mmol}, \mathrm{c}=0.04 \mathrm{M}$, if not otherwise stated) in the stated solvent was pumped through a glass column (Omnifit ${ }^{\circledR}$, $10.0 \mathrm{~mm}$ i.d. $\times 100.0 \mathrm{~mm}$ length) packed with the solid supported reagent (1.8 $\mathrm{g}$ for silica-supported $\mathrm{BF}_{3}, 0.8 \mathrm{~g}$ for all other solid-supported reagents) and heated to the stated temperature. A 100 psi back pressure regulator was placed after the reactor. The solution obtained was concentrated in vacuo. To isolate the free amine the solution was washed with saturated $\mathrm{NaHCO}_{3}$ solution $(10 \mathrm{~mL})$ prior to concentration.

\section{Supplementary Material}

Detailed experimental procedures and characterization data are available on the Journal's website.

\section{Acknowledgement}

We gratefully acknowledge the EPSRC (Award No. EP/K009494/1) for funding.

\section{References}

[1] S. Saaby, K. R. Knudsen, M. Ladlow, S. V. Ley, Chem. Commun. 2005, 2909-2911.

[2] C. J. Smith, C. D. Smith, N. Nikbin, S. V. Ley, I. R. Baxendale, Org. Biomol. Chem. 2011, 1927-1937.

[3] A. G. O’Brien, F. Lévesque, Y. Suzuki, P. H. Seeberger, Chim. Oggi 2011, 29, 57-60.

[4] P. Poechlauer, S. Braune, B. Dielemans, B. Kaptein, R. Obermüller, M. Thathagar, Chim. Oggi 2012, 30, 5154.

[5] L. Vaccaro, D. Lanari, A. Marrocchi, G. Strappaveccia, Green Chem. 2014, 16, 3680-3704 (and references cited therein).

[6] I. R. Baxendale, C.-M. Griffith-Jones, S. V. Ley, G. Tranmer, Chem. Eur. J. 2006, 12, 4407-4416.

[7] T. Razzaq, T. N. Glasnov, C. O. Kappe, Chem. Eng. Technol. 2009, 32, 1702-1716.

[8] T. Razzaq, C. O. Kappe, Chem. Asian J. 2010, 5, 1274-1289.

[9] D. L. Browne, B. H. Harji, S. V. Ley, Chem. Eng. Technol. 2013, 36, 959-967.

[10] A. Sugimoto, T. Fukuyama, M. T. Rahman, I. Ryu, Tetrahedron Lett. 2009, 50, 6364-6367.

[11] M. Rasheed, T. Wirth, Chim. Oggi 2011, 3, 54-56.

[12] M. Rueping, T. Bootwicha, E. Sugiono, Beilstein J. Org. Chem. 2012, 8, 300-307.

[13] C. F. Carter, H. Lange, S. V. Ley, I. R. Baxendale, B. Wittkamp, J. G. Goode, N. L. Gaunt, Org. Process. Res. Dev. 2010, 14, 393-404.
[14] D. L. Browne, S. Wright, B. J. Deadman, S. Dunnage, I. R. Baxendale, R. M. Turner, S. V. Ley, Rapid Commun. Mass Spectrom. 2012, 26, 1999-2010.

[15] I. R. Baxendale, L. Brocken, C. J. Mallia, Green Process Synth. 2013, 2, 211-230.

[16] F. Lévesque, P. H. Seeberger, Angew. Chem. 2012 , 124, 1738-1741; Angew. Chem. Int. Ed. 2012, 51, 17061709.

[17] S. Newton, C. F. Carter, C. M. Pearson, L. de C. Alves, H. Lange, P. Thansandote, S. V. Ley, Angew. Chem. 2014, 126, 5015-5020; Angew. Chem. Int. Ed. 2014, 53, 4915-4920.

[18] For a recent review see: J. C. Pastre, D.L. Browne, S.V. Ley, Chem. Soc. Rev. 2013, 42, 8801-9198.

[19] V. A. Soloshonok, H. T. Catt, T. Ono, J. Fluorine Chem. 2009, 130, 512-515.

[20] R. Marion, G. Muthusamy, F. Geneste, J. Catalysis 2012, 286, 266-272.

[21] M. A. Kabeshov, B. Musio, P. R. D. Murray, D. L. Browne, S. V. Ley, Org. Lett. 2014, 16, 4618-4621.

[22] J. L. Pousset, J. Kerharo, G. Maynart, A. Cavé, R. Goutarel, Phytochemistry 1973, 12, 2308-2310.

[23] J. L. Pousset, A. Cavé, A. Chiaroni, C. Riche, J. Chem. Soc., Chem. Commun. 1977, 261-262.

[24] F. Tillequin, M. Koch, J. Chem. Soc., Chem. Commun. 1978, 826-828.

[25] A. N. Ratnagirisvvarnn, K. Venkatachalam, J. Indian Chem. Soc. 1942, 19, 389-392.

[26] K. K. Purushothaman, A. Sarada, Phytochemistry 1981, 20, 351-352.

[27] A.M. Baldé, L. A. Pieters, A. Gergely, V. Wray, M. Claeys, A. J. Vlietinck, Phytochemistry 1991, 30, 9971000.

[28] L. S. Fernandez, M. F. Jobling, K. T. Andrew, V. M. Avery, Phytother. Res. 2008, 22, 1409-1412.

[29] L. S. Fernandez, M. S. Buchanan, A. R. Carroll, Y. J. Feng, R. J. Quinn, V. M. Avery, Org. Lett. 2009, 11, 329332.

[30] L. S. Fernandez, M. L. Sykes, K. T. Andrew, V. M. Avery, Int. J. Antimicrob. Agents 2010, 36, 275-279

[31] D. H. Dethe, R. D. Erande, A. Ranjan, J. Am. Chem. Soc. 2011, 133, 2864-2867

[32] R. M. Zeldin, F. D. Toste, Chem. Sci. 2011, 2, 17061709.

[33] R. Vallakati, J. A. May, J. Am. Chem. Soc. 2012, 134, 6936-6939.

[34] R. Vallakati, J. A. May, Synlett 2012, 23, 2577-2581. [31] D. H. Dethe, R. D. Erande, A. Ranjan, J. Org. Chem. 2013, 78, 10106-10120.

[35] R. Vallakati, J. P. Smuts, D. W. Armstrong, J. A. May, Tetrahedron Lett. 2013, 54, 5892-5894.

[36] D. H. Dethe, R. D. Erande, B. D. Dherange, Org. Lett. 2014, 16, 2764-2767.

[37] See Supplementary Material for details of the flow setup used for the flow experiments.

[38] M. Bandini, M. Fagioli, A. Melloni, A. UmaniRonchi, Adv. Synth. Catal. 2004, 346, 573-578.

[39] PVPP $-\mathrm{BF}_{3}=$ Polyvinylpyrrolidon tetrafluoroborate [40] M. M. Lakouraj, M. Mokhtary, Monatsh. Chem. 2009, 140, 53-56. 
[41] C. R. Quinn, J. H. Clark, S. J. Tavener, K. Wilson, Green Chem. 2003, 5, 602-605.

[42] Determined by ${ }^{1} \mathrm{H}$ NMR (400 MHz, MeOH- $d^{4}$ ): $\delta=$ 4.34 (q, $J=8.7 \mathrm{~Hz})$ vs. $4.25(\mathrm{q}, J=8.5 \mathrm{~Hz})$.

[43] L. Guetzoyan, R. J. Ingham, N. Nikbin, J. Rossignol, M. Wolling, M. Baumert, N. A. Burgess-Brown, C. M. Strain-Damerell, L. Shrestha, P. E. Brennan, O. Fedorov, S. Knapp, S. V. Ley, Med. Chem. Commun. 2014, 5, 540546. 